Authors

Roberto William Invernizzi Department of Neuroscience, Mario Negri Institute for Pharmacological Research, Milan, Italy

Ennio Esposito Laboratory of Neurophysiology, Consorzio "Mario Negri" Sud, Santa Maria Imbaro (Chieti), Italy

Dr. Vincenzo Di Matteo Istituto di Ricerche Farmacologiche "Mario Negri", Santa Maria Imbaro, Italy

- Professor Giuseppe Di

Giovanni

Department of Physiology and Biochemistry, Laboratory for the Study of Neurological for the Study of
Disorders, Malta

Editors

Prof. Ian P. Stolerman Department of Addictions, Institute of Psychiatry P048, London SE5 8AF, UK

8 L

awrence $\mathrm{H}$. Price The Warren Alpert Medical School of Brown University, Providence, USA

Session History (max. 10)

Microiontophoresis and Related Methods

Giuseppe Di Giovanni

Index of Editors and Authors Ennio Esposito
Biomedical and Life Sciences > Encyclopedia of Psychopharmacology > Microiontophoresis and Related Methods

Cite | History | Comment | Print | References | Image Gallery | Hide Links

Update Article

Logout

\section{Microiontophoresis and Related Methods}

\section{Synonyms}

Iontophoresis

\section{Definition}

The term microiontophoresis is derived from the ancient Greek term phoretikos, which refers to the production or induction of movement. Microiontophoresis is a technique with which drugs and other ionized particles can be ejected in very small amounts from solutions contained in glass micropipettes. This ejection is accomplished by applying a voltage across the micropipette and causing the electrode to become polarized. Ionized particles in solution migrate in the applied field and will be ejected from the tip as they carry the current into the tissue. This technique is widely used to determine the effects of various substances on firing parameters of both central and peripheral neurons and muscles. In investigating the phenomenon of synaptic transmission at the neuromuscular junction, during the 1950s, this technique became very popular. A technique appropriate for the study of synaptic pharmacology was first realized by Nastuk (1953) and was later developed by del Castillo and Katz (1955), and it consisted essentially of the microiontophoretic method, i.e., movement of charged particles produced by an electric current, restricted to a micropipette with a tip diameter of the order of $1 \mu \mathrm{m}$. Thus, solutions of acetylcholine chloride were used, and by passing a suitable current to this solution, acetylcholine ions could be ejected from the $1 \mu \mathrm{m}$ orifice onto a correspondingly localized area of subsynaptic membrane at the neuromuscular junction. Later, Curtis and his colleagues adopted this technique for studying the mammalian central nervous system (CNS) (Curtis and Eccles 1958b). The experiments of Curtis and coworkers, however, involved an important modification of the original method, in that this group used multibarrel micropipettes. In the production of these, several lengths of tubing are fused together and then pulled so as to produce a single collective tip, but with each barrel having its own orifice. Multibarrel micropipettes are usually composed of five to seven barrels (Fig. 1). Usually, the central barrel is the recording electrode, whereas the other side barrels contain drug solutions (Fig. 1). As the drug molecules would tend to diffuse from solution in the pipette tip into the extracellular environment, it is necessary to apply a small current to reduce that efflux. This is known as a "holding" or "retaining" current (Fig. 2). It is also a usual practice to include a barrel containing sodium chloride solution, which can be used to control the effects of the current itself. This may be done either by periodically passing through the control barrel the same current used for drug ejection or by passing continuously a current adequate to cancel out the instantaneous sum of ejecting and retaining currents passing through the drug-containing barrels. This is known as "current balancing." The use of microiontophoresis is suitable for any ionized molecule, but nonionized compounds can be ejected by the closely related variant "electro-osmosis," which is attributable to the presence of an electrical "double layer" within the barrel tip. When an aqueous solution is in contact with glass, negative ions are tightly adsorbed on the glass surface, leaving the bulk of solution carrying a net positive charge. The passage of positive (or outward) current then causes the ejection of a small volume of solution containing the compound interest (Fig. 2). It should be noted, however, that this mechanism has nothing to do ware the osmotic pressure of a solution or the establishment of any osmotic gradient. The term electro-osmosis derives simply from the fact that the driving force is the movement of the solvent, not the solute, just as the case of osmotic movements across a semipermeable membrane. An alternative method of applying both ionized and nonionized compounds from micropipettes is the use of pressure. A suitable source of pressure, usually a cylinder of compressed gas, is connected to the open end of a micropipette barrel. Pressure usually up to 20 pounds per square inch (p.s.i.) will eject fluid from a $1 \mu \mathrm{m}$ pipette tip. One advantage of micropressure ejection is that it can be applicable to all compounds; however, it is not devoid of problems and artifacts and is unlikely to replace microiontophoresis as a microapplication method. 


\section{Microiontophoresis and Related Methods}

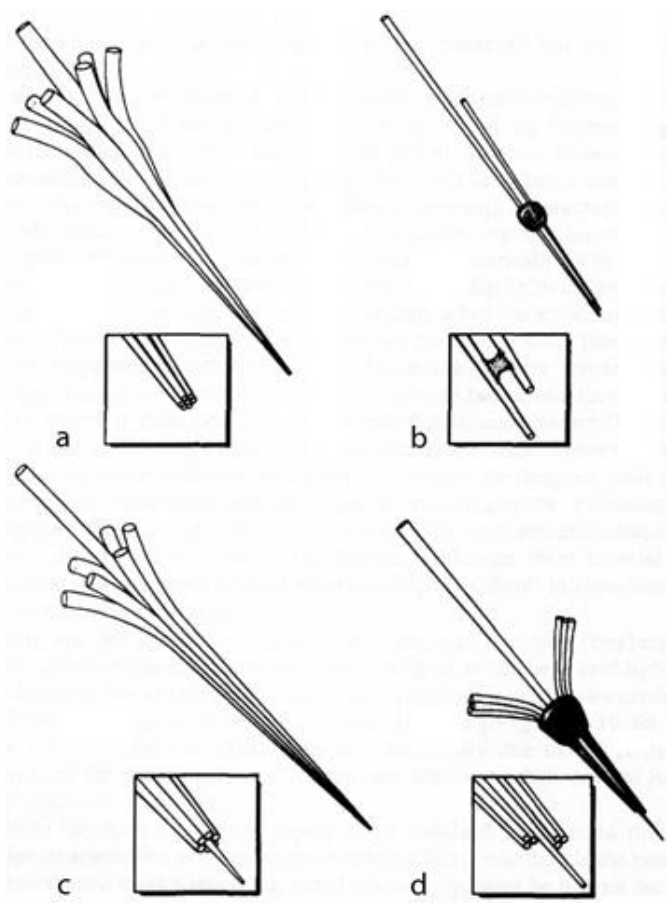

Microiontophoresis and Related Methods. Fig. 1. Examples of different types of multibarrel micropipette assemblies used in microiontophoretic experiments. (a) Standard 7-barrel assembly in most common use, introduced first by Curtis. (b) Twin, or parallel micropipette. (c) Co-axial assembly. (d) Staggered tip multibarrel. (From Hicks, 1984.)

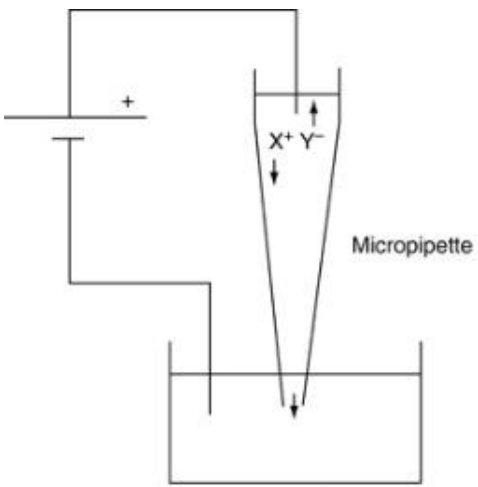

Ejecting $x^{*}$

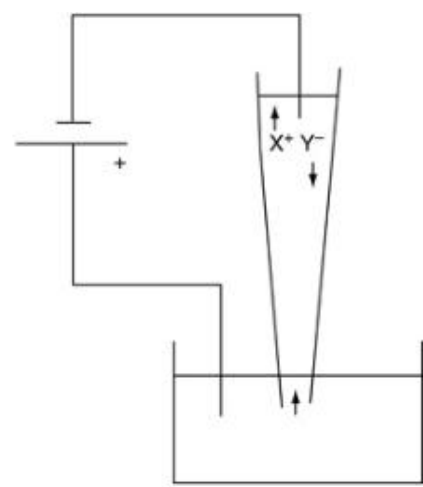

Retaining $x^{+}$

Microiontophoresis and Related Methods. Fig. 2. Schematic diagram of a micropipette that contains a salt $\mathrm{X}^{+} \mathrm{Y}^{-}$, showing the direction of current necessary to eject (a) and retain (b) the ion $\mathrm{X}^{+}$. (From Hicks, 1984.)

\section{Principles and Role in Psychopharmacology}

\section{General Principles}

Each barrel of a micropipette assembly to be used for drug ejection is filled with a solution of the ionized compound and the solution is connected to the iontophoresis machine by a suitable lead, which is in contact with the drug solutions. The establishment of a potential difference between the drug solution and the medium surrounding the barrel tip will then cause the movement of ions through the solution and out of the pipette tip (Fig. 2). A chief advantage of the microiontophoretic method is that it is possible to examine the effects of drugs on single neurons in vivo without affecting the whole nervous system or other physiological responses, such as those that may occur when drugs are administered systemically (Aghajanian 1972). If a voltage is applied to a solution, ions and charged 


\section{Microiontophoresis and Related Methods}

molecules will migrate toward and away from the source of the imposed electrical field depending on the sign of their net charge. This phenomenon is the fundamental principle of microiontophoresis: the desired charged particles are ejected from the mouth of one barrel of a multipipette assembly by appropriately charging the interior of that barrel (Fig. 2). An outward current will cause the "ejection" of positively charged ions, and an inward current flow, the ejection of negatively charged particles. If the pipette assembly is positioned close to a neuron, so that the recordings of its activity can be made through another electrolyte-filled barrel, drugs may be ejected and their pharmacological effects are inferred by the resulting changes in the rate and/or firing pattern.

\section{The Transport Number and the $\mathrm{T} 50$ Value}

An important technical consideration for experiments employing microiontophoresis is the transport number. The transport number is a measure of the amount of drug released from the micropipette by iontophoretic expulsion and it is important, because it helps one to evaluate dose-response relations between different compounds, and it can also provide some indication of the absolute potency of compounds. The transport number varies for individual compounds and is based on the interaction of the following variables: their solubility, the extent of their dissociation in solution, their polarity, and the nature of the external medium into which the drugs are administered. The transport number may be formally described by the following equation:

$n=R_{i} \mathrm{ZFi}^{-1}$, where

$n=$ apparent transport number of the drug ion,

$Z$ = valency,

$F=$ Faraday's constant, in Coulombs

$i=$ intensity of ejecting current, in nanoamperes, and

$R i=$ rate of microiontophoretic release (which is equivalent to total release minus the sum of the rate of steady-state spontaneous release and where applicable, the release due to electro-osmosis).

During microiontophoresis, the total number of ions transported is related in a direct manner to the amount of current applied to the solution, according to Faraday's law. However, only a certain proportion of the charge imposed is carried by the ion species of interest. This value, which is " $n$," the transport number, is not constant for a given material but will vary not only from pipette to pipette, but also, to a lesser extent, between different barrels of the same micropipette assembly containing identical solutions. Despite these inconsistencies, it remains valid that under steady-state conditions, drug release from micropipettes conforms to Faraday's law: the amount of drug released is proportional to the magnitude of current passed (Hicks 1984).

Another important parameter to consider when interpreting microiontophoretic data is the $T$ 50 value, which is the time taken for a response to reach $50 \%$ of its maximum (Fig. 3). The basis for this procedure is the hypothesis that each individual response to an agonist may be considered as a cumulative dose-response relationship reflecting the gradual increase in tissue concentration of drug during the ejection period. If a series of such responses are obtained, reaching the same maximum amplitude, they can be readily characterized by the $T 50$ value (Fig. 3). Moreover, $T 50$ value is easier to measure accurately than a response size. Thus, it can be very difficult to obtain reproducible graded response amplitudes to some very potent compounds such as amino acids. Any changes in firing rate during a response, which ideally should be of the plateau variety, may further complicate any assessment of the response size, whereas in the determination of $T 50$ values, all responses increase to the same maximal level, which may be $100 \%$ inhibition or a clear maximal plateau of excitation tending toward overdepolarization.

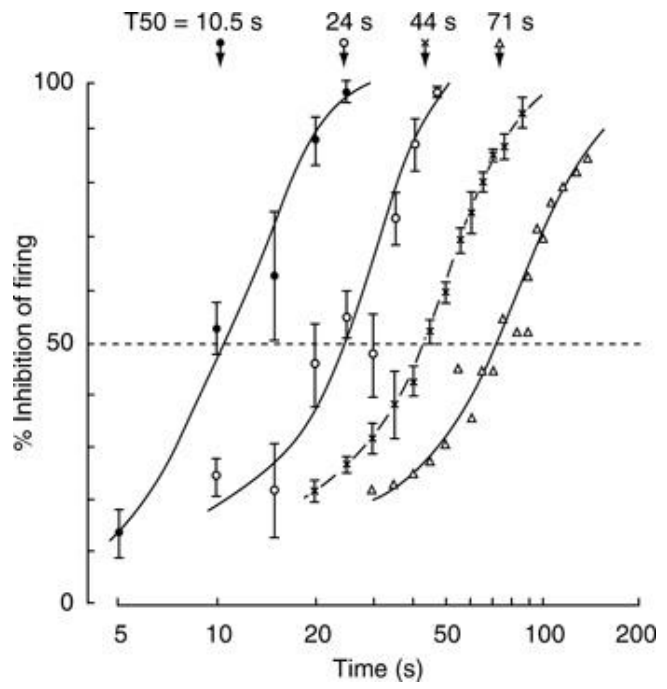

Microiontophoresis and Related Methods. Fig. 3. Time courses of inhibition of neuronal firing following microiontophoretic application of GABA with four different currents, $20 \mathrm{nA}$ (filled circle), $10 \mathrm{nA}(0), 5 \mathrm{nA}(\times)$, and $2 \mathrm{nA}$ (open triangle). Each curve was obtained from the same neuron at a depth of $957 \mu \mathrm{m}$ in the middle suprasylvian gyrus of the cat cortex. The neuron was driven by continuous microiontophoretic application of L-glutamate (20 nA). Each of the points for 20, 10, and $5 \mathrm{nA}$ applications of GABA is the mean \pm SEM of three values obtained from three separate applications of the same current of GABA. The values of T50 shown 


\section{Microiontophoresis in the Central Nervous System}

It is now more than 50 years since Curtis and Eccles $(1958 a, b)$ first employed the technique of microiontophoresis in the CNS. Microiontophoresis has provided so far a great contribution in the identification of the central effects of neurotransmitters, including glutamate, aspartate, $y$-aminobutyric acid (GABA), noradrenaline, serotonin, dopamine, and a variety of neuropeptides (enkephalins, cholecystokinin, neurotensin, tachykinins). Microiontophoresis also allows the histological confirmation of the sites of electrophysiological recordings, and the neuroanatomical determination of pathways by applying dyes, markers, and materials, which are carried by axonal transport for tracing fiber tracts. Alterations in neuronal sensitivity due to the influence of anesthetic compounds have been monitored when pharmacological agonists have been tested using microiontophoresis.

\section{Applications}

The largest number of studies has been concerned with the central nervous system. These studies have yielded information on: (1) the qualitative sensitivity of neurons to putative neurotransmitters and drugs; (2) quantitative estimates of variations and sensitivity in different CNS regions or of different cell types and the following lesions or the administration of drugs; (3) the pharmacology of transmitter receptors; (4) the effects of modifier of putative transmitter effects (antagonistic or enhancing substances) on synaptic transmission; and (5) the mechanisms and ionic conductances underlying transmitters effects.

\section{Excitatory Amino Acids}

Some of the earliest iontophoretic studies demonstrated marked excitatory activity of several simple dicarboxylic acids, including L-glutamic and L-aspartic acids (Curtis and Watkins 1960). Responses to some of these amino acids, especially glutamate and aspartate, terminate rapidly when an ejecting iontophoretic current is switched off. It is unclear to what extent this is due to the kinetics of iontophoresis or reflects the presence of rapid and efficient uptake processes. Some authors have reported long-lasting changes of cortical neuronal firing following iontophoresis of glutamate sufficient to at least double the resting firing rate. The development of a series of potent amino acids analogs with very high agonist potency led to the discovery of NMDA and non-NMDA glutamate receptors. This discovery was strengthened by additional findings that phosphonate analogs of amino acids, such as 2-amino-5-phosphonovaleric acid (AP-5) blocked the effects of NMDA, but not of quisqualic and kainic acids.

\section{Inhibitory Amino Acids}

Both glycine and GABA act as potent inhibitors of neuronal activity in the CNS, usually causing hyperpolarization associated with increased membrane conductance to chloride. Glycine is selectively antagonized by strychnine, whereas the effects of GABA are blocked by picrotoxin and bicuculline. Microiontophoretic experiments showing potentiation of the inhibitory effects of GABA by benzodiazepines were among the earlier experimental evidence for the modulatory action of these drugs on GABAA receptors (Gallagher 1978).

\section{Acetylcholine}

Almost every region of the brain has been examined for its sensitivity to iontophoretically applied cholinergic agents. Most of the earlier work in vivo was concerned primarily with establishing the direction of responses to cholinomimetics and whether the effects involved muscarinic or nicotinic receptors. Many studies examined only cells encountered randomly in a particular brain region, but others have often succeeded in relating the direction of responses to cholinomimetics with some specific function. In the cerebral cortex deep pyramidal tract, cells are excited by acetylcholine. Several authors have also described an inhibitory action of acetylcholine, largely muscarinic in nature, in more superficial levels of the cortex and an excitatory action, which appears to have a predominant nicotinic pharmacology, in the same superficial layers. Some authors have shown that acetylcholine enhances the stimulus-evoked responses of visually driven cortical units, without affecting the overall excitability of the cell. Thus, orientation and direction specificity of neurons is preserved and increased relative to the nonpreferred responses. This phenomenon is reminiscent of the effects of some amines, which can also increase the signal-to-noise ratio by potentiating evoked activity and suppressing background.

\section{Noradrenaline}

Early microiontophoretic studies have shown that noradrenaline would cause a depression of neuronal firing in the cat cerebral cortex, and a large number of experiments have revealed similar responses in most areas of the CNS. This inhibition often seems to involve a voltage-dependent hyperpolarization accompanied by an increased membrane resistance, although a decreased membrane resistance was found on neurons of the locus coeruleus in slice preparation in vitro. The biochemical basis of this hyperpolarization has been the subject of much argument. Although it was originally suggested that they may be mediated by an increase in the intracellular concentration of cyclic AMP, some group failed to reproduce these findings. Overt excitatory effects of noradrenaline have also been observed in many areas of the CNS. Neuronal responses to iontophoretic application of noradrenaline, apparently excitatory as well as inhibitory, can be enhanced by antidepressants. However, this potentiation can occur even after the loss of most aminecontaining terminals, and it may be restricted to certain layers of the cortex. The pharmacology of responses to iontophoretically applied noradrenaline has been extensively studied. Some authors have postulated that, in the neocortex, excitatory responses to noradrenaline are mediated by $a_{1}$-adrenergic receptors, whereas inhibitory responses occur through $\beta$-adrenergic receptors. Activation of $a_{2}$-adrenergic receptors does also elicit inhibitory responses.

\section{Dopamine}

Dopamine was first tested iontophoretically in the cerebral cortex, where profound suppression of spontaneous cell firing was observed. This action has been confirmed by several authors, although excitatory effects have also been reported. Much attention has 
been centered on the effects of dopamine in the neostriatum where its action is usually inhibitory in the caudate nucleus. Bunney and Aghajanian (1976) have performed a laminar analysis of amine responses in the rat cerebral cortex. They found that neurons in layers II and III, which receive a dense noradrenergic projection, were more sensitive to noradrenaline than dopamine, whereas the reverse pattern was noted in layers $\mathrm{V}$ and VI, which receive a greater dopamine-containing projection. These authors also reported that desipramine, a selective inhibitor of noradrenaline reuptake, would enhance noradrenaline responses in layers II and III, but not in deeper layers, while benztropine enhanced dopamine responses only in layers V and VI. Dopamine receptors are present not only on innervated cells but also on the dopaminergic neurons themselves: the so-called autoreceptors. Activation of such receptors by dopamine or apomorphine causes marked inhibition of cell firing, and these effects are blocked by neuroleptic drugs.

Microiontophoretic studies of dopamine response pharmacology have mostly proved consistent with behavioral and neurochemical work. Phenothiazines, for example, block dopamine but not noradrenaline responses in the cerebral cortex and the striatum. Iontophoretically applied a-flupenthixol can also block the effects of dopamine, although intravenously administered a-flupenthixol or pimozide did not modify neuronal responses to iontophoretic dopamine.

\section{Serotonin}

There is an extensive scientific literature regarding the effects of microiontophoretically applied serotonin on different areas of the central nervous system. Indeed, the microiontophoretic technique contributed substantially to the elucidation of the physiology and pharmacology of the central serotonergic system. Thus, an important factor controlling the activity of central serotonergic neurons is neuronal feedback inhibition. This is thought to be a homeostatic response, which, under physiological conditions, acts to compensate for increases in synaptic availability of serotonin. Thus, as the concentration of serotonin increases in the brain, the activity of central serotonergic neurons correspondingly decreases. The mechanism underlying this feedback regulation is both local or intrinsic to the raphe region (where serotonergic cell bodies are located) and through a feedback loop from postsynaptic target neurons. Serotonin released in the raphe region from dendrites and possibly from axon terminals appears to inhibit serotonergic neurons by activating somatodendritic autoreceptors, which produces hyperpolarization of the cell membrane via an increase in potassium conductance. Historically, the first drug reported to exert a preferential action on the 5-HT autoreceptor was LSD (Iysergic acid diethylamide) applied microiontophoretically on the dorsal raphe nucleus of rats. Subsequently, several other hallucinogenic indoleamines, notably 5-MeODMT (5-methoxy- $N, N$-dimethyltryptamine), were found to share this property with LSD. Since that time, several highly selective $5-\mathrm{HT}_{1} \mathrm{~A}$ agonist compounds such as $8-\mathrm{OH}$-DPAT have been synthesized and shown to suppress the firing of serotonergic neurons with potencies comparable with, or even greater than, that of LSD. On the basis of electrophysiological data, the serotonin autoreceptor has been characterized as the $5-\mathrm{HT}_{1} \mathrm{~A}$ subtype. Microiontophoretic technique also contributed to characterize the action of serotonin agonists and antagonists and to elucidate the physiological role of serotonin receptor subtypes such as $5-\mathrm{HT}_{1} \mathrm{~B}, 5-\mathrm{HT}_{2} \mathrm{~A}$, and $5-\mathrm{HT}_{2} \mathrm{C}$. As regards the $5-\mathrm{HT}_{2} \mathrm{C}$, it was found that this receptor subtype exerts a tonic inhibitory influence on the activity of dopamine-containing neurons in the substantia nigra pars compacta and the ventral tegmental area. Apparently, this inhibitory effect is mediated through the activation of nondopaminergic (presumably GABA-ergic) neurons in the substantia nigra pars reticulata. Thus, it was recently shown that microiontophoretic application of $5-\mathrm{HT}_{2} \mathrm{C}$ receptor agonists stimulates the basal activity of nondopaminergic (presumably GABA-ergic) neurons in the substantia nigra pars reticulata (Invernizzi et al. 2007) (Fig. 4). By using microiontophoresis, it was also found that serotonin exerts a tonic inhibitory influence on the activity of noradrenergic neurons in the locus coeruleus.

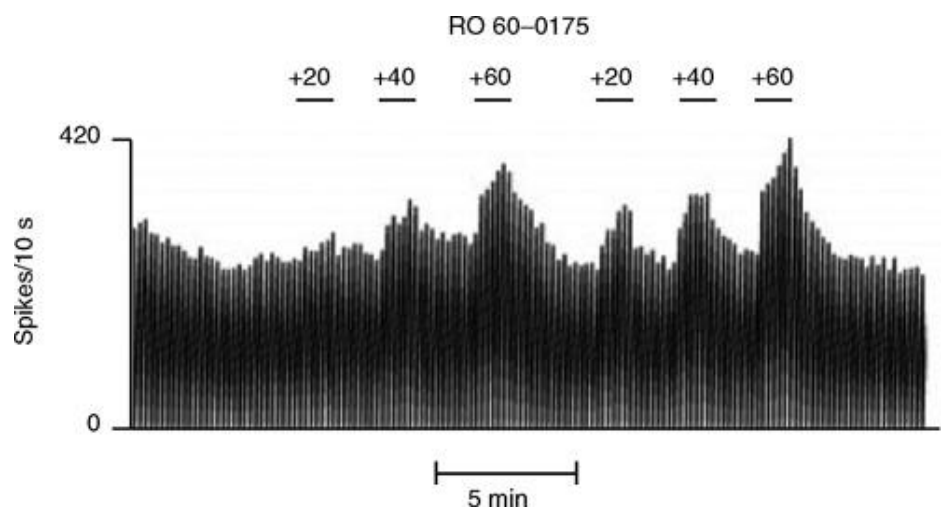

Microiontophoresis and Related Methods. Fig. 4. Representative rate histogram showing the effect of the selective $5-\mathrm{HT}_{2} \mathrm{C}$ receptor agonist $\mathrm{RO} 60-0175$ on the basal activity of a nondopaminergic (presumably GABA-ergic neuron) of the rat substantia nigra pars reticulata. Microiontophoretic application of RO 60175 causes excitation of basal neuronal activity, which is proportional to the amount of the ejecting current applied (numbers above each bar in $\mathrm{nA}$ ). (From Invernizzi et al. 2007.)

\section{Opiates and Opioids}

Microiontophoresis has proved exceedingly valuable for opiate system studies, since it allows the testing of discrete units activated by noxious or nonnoxious stimuli in the same preparation. In most such studies, the applied opiates have depressed noxious stimulusevoked activity, although usually in parallel with the effects on spontaneous or chemically 


\title{
Microiontophoresis and Related Methods
}

induced firing. Microiontophoresis has also been proved as a popular means for comparing qualitatively opiate responses in normal and opiate-tolerant animals. Thus, inhibitory responses to morphine were encountered less frequently in the neocortex of morphinetolerant rats than in controls. It was shown that iontophoretically applied naloxone would elicit a large increase of firing in the locus coeruleus noradrenergic neurons in morphinetolerant rats, presumably as a correlate of the withdrawal phenomenon in such animals. Also, opioid peptides have been tested iontophoretically in many regions of the central nervous system. Opioid peptides were found to excite hippocampal neurons; however, these effects were apparently mediated through an indirect action on transmitter release or to a naloxone-sensitive depression of local inhibitory interneurons.

\section{Peptides}

Microiontophoretic or pressure ejection has been used to apply a wide range of endogenous and synthetic peptides to neurons in vivo and in vitro. However, partly because of the lack of selective antagonists, there has been little progress in relating the observed responses to a physiological role, and as a result, attention has been concentrated on the mechanism of the observed responses, and potential interactions with neurotransmitters. Substance $P$, for example, appears to interact selectively with acetylcholine. Microiontophoretic substance $\mathrm{P}$ has also been found to enhance the response of spinal cord neurons to noxious stimulation but not innocuous ones, in some cases leading to the occurrence of responses in initially unresponsive units. Some excitatory effects of substance P can be mimicked by capsaicin, also applied iontophoretically. It was also reported that the excitatory effect of substance $P$ on noradrenalin-containing neurons in the locus coeruleus is blocked by the selective antagonist $\left[\mathrm{D}-\mathrm{Pro}^{2}, \mathrm{D}-\mathrm{Trp}^{7,9}\right]$ substance P. Thyrotropin releasing hormone (TRH) has been found to enhance the excitatory effects of acetylcholine on cortical neurons, with no effects on resting firing rate. Somatostatin exerts a potent excitatory effect on hippocampal neurons. Cholecystokinin (CCK) and neurotensin are also frequently excitatory while angiotensin has excitant properties, which appear to be restricted to the subfornical organ and related structures. However, it is important to point out that peptides present special problems for microiontophoresis. Larger molecules tend to be adsorbed on to charged surfaces, which include the internal wall of a micropipette tip. Some peptides may also undergo denaturation or degradation during iontophoretic experiments. This problem may be exacerbated if very high currents are applied for long periods of time through high resistance tips, in that any change of local temperature may have a major impact on the stability of a peptide.

\section{Advantages and Disadvantages of Microiontophoresis}

The original microiontophoretic technique was developed for answering questions concerned with synaptic transmission and the neuromuscular junction. Using this preparation, it is a simple matter to microscopically examine the muscle fiber being studied, to determine the distance of the micropipette from the tissue, and to have ready access to known synaptic inputs. These advantages are not valid for the CNS. Nevertheless, with some further precautions and considerations, the technique has been used successfully in the CNS for about 50 years. It is important to consider other potentially confounding technical factors limiting the utility of microiontophoresis, as it is used in central investigations. Of primary concern is the site of drug administration relative to cell soma, where the strongest depolarizing or hyperpolarizing influences are manifested, and the dendritic field, where synaptic influences are normally expressed and where antagonists of transmitters must accumulate to modify trans-synaptic excitations. Another consideration for central investigations also concerns the spatial distribution of drugs in the CNS. Since the CNS is densely packed with cells, microiontophoretically administered compounds cannot affect single neurons in isolation. This must be kept in mind when interpreting the data.

\section{Conclusions}

Microiontophoresis, an experimental technique introduced more than 50 years ago, prompted a great impetus to the study of the physiology and pharmacology of the central nervous system. By mimicking the synaptic function, it provided a crucial step in establishing the physiological role of most neurotransmitters, including amines, amino acids, and neuropeptides. Although it is now considered by some as a "classical" neurophysiologic approach to the study of central nervous system, it is likely that it will still contribute substantially to the progress of neuroscience.

\section{Cross-References}

\author{
Antidepressants \\ Excitatory Amino Acids \\ Extracellular Recording \\ Hallucinogens \\ Inhibitory Amino Acids \\ Intracellular Recording \\ Neurotensin \\ Opioids \\ Somatostatin \\ Tachykinins
}

\section{References}

Aghajanian GK (1972) LSD and CNS transmission. Annu Rev Pharmacol 12:157-168

Bunney BS, Aghajanian GK (1976) Dopamine and norepinephrine innervated cells in the rat prefrontal cortex: pharmacological differerentiation using microiontophoretic techniques. Life Sci 19:1783-1789

Curtis DR, Eccles RM (1958a) The excitation of Renshaw cells by pharmacological agents applied electrophoretically. J Physiol 141:435-445

Curtis DR, Eccles RM (1958b) The effect of diffusional barriers upon the pharmacology of cells within the central nervous system. J Physiol 141:446-463 


\section{Microiontophoresis and Related Methods}

Curtis DR, Watkins JC (1960) The excitation and depression of spinal neurones by structurally related amino acids. J Neurochem 6:117-141

del Castillo J, Katz B (1955) On the localization of acetylcholine receptors. J Physiol 128:157-181

Gallager DW (1978) Benzodiazepines: potentiation of a GABA inhibitory response in the dorsal raphe nucleus. Eur J Pharmacol 49:133-143

Hicks TP (1984) The history of development of microiontophoresis in experimental neurobiology. Prog Neurobiol 22:185-240

Invernizzi RW, Pierucci M, Calcagno E, Di Giovanni G, Di Matteo V, Benigno A, Esposito E (2007) Selective activation of $5-\mathrm{HT}_{2} \mathrm{C}$ receptors stimulates GABA-ergic function in the rat substantia nigra pars reticulata: a combined in vivo electrophysiological and neurochemical study. Neuroscience 144:1523-1535

Nastuk WL (1953) Membrane potential changes at a single endplate produced by transitory application of acetylcholine with an electrically controlled microjet. Fed Proc 12:102 